\title{
Soft Materials used in heart valve research: A critical review
}

\author{
Abhimanyu Tharayil, ${ }^{1}$ Vivek Verma, ${ }^{1}$ Malay K. Das, ${ }^{2}$ and Kamal K. Kar ${ }^{2,3 *}$ \\ ${ }^{1}$ Department of Materials Science and Engineering, ${ }^{2}$ Department of Mechanical Engineering, \\ ${ }^{3}$ Advanced Nanoengineering Materials laboratory, Materials Science Programme, \\ Indian Institute of Technology Kanpur, Kanpur-208016, India \\ *Email:kamalkk@iitk.ac.in
}

The human heart is a very complex organ and for the human heart to work properly there should be unidirectional flow of blood, and it is the heart valves which ensures it. Each heart valve has a set of flaps called leaflets. Sometimes leaflets does not open as wide as it should, because of the thickening or fusing up, and as a result the smooth unidirectional flow of blood is hindered. In order to fix these problems, surgeons either repair the valve or replace it. Replacement valves can be of bioprosthetic or tissue valves and mechanical heart valves. The latter have long-term durability but lifelong anticoagulation is required with high risk of thrombosis. Biological valves are usually constructed from pig or cow tissues and are specially treated so that the body does not reject them. However, they have a smaller lifetime compared to their mechanical counterparts [1].

Polymeric materials are amongst the most studied valve materials, and as such flexible polyurethanes and silicone rubbers were manufactured long back around 1950s to be used as valve materials. It was expected to mimic the natural valve due to its elastic and flexible nature but were found to be less durable than their mechanical counterparts and the instances of tearing, and calcification were profound. The choice of material is the most important and crucial parameter in the development of heart valve materials. For a polymeric heart valve to be practically used it should adhere to the acceptable standards of biostability, hemocompatibility, resistance to both degradation and calcification etc. These stringent factors reduce the usability of many polymers. Polyurethane (PU) is one of the most commonly used polymer in heart valve prosthesis. Segmented polyurethanes were developed which had both hard and soft segments, thereby providing elastic as well as strong mechanical properties. Substitution chemistry has been done to enhance the biocompatibility of polyurethane's. The tilting disc in the TTK Chitra valve (considered the cheapest mechanical valve) is of Ultra high molecular weight polyethylene.

Very recently hyaluronan with polyethylene films have been used as a clip, in surgical instruments and showed very less blood clotting [4]. Figure 1 [3] gives an overview of the chronological use of different polymers as valve materials over time. Reinforcing the polymer with fibers have been done to increase the mechanical properties of the polymer. Surface modifications at the nanoscale can also be done to improve the selective surface properties for example biostability of the polymeric materials without altering their bulk properties.

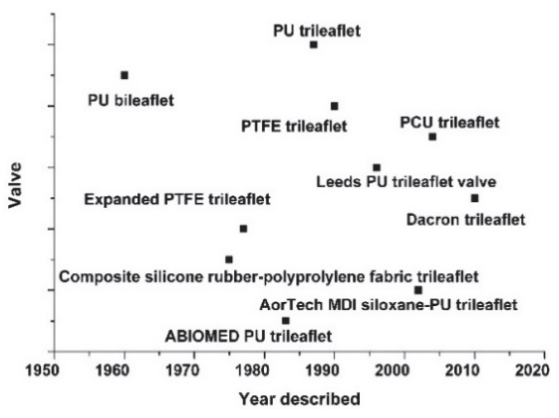

Figure 1: Chronological use of different polymer material as heart valve through years

\section{References}

1. Yacoub MH, Cohn LH. Circulation 2004; 109:942-950

2. Lamba N, Woodhouse K, Cooper S: Boca Raton: CRC Press; 1997.

3. Claiborne, T. E.; Slepian, M. J.; Hossainy, S.; Bluestein, D. Expert Rev. Med. Devices 2012, 577 $-594$

4. D.A Prawel, H. Dean, M. Forleo, N. Lewis,J.Gangwish, K.C.Popat Cardiovasc Eng Technol, 5 (2014), pp 70-81. 